Supplement of Nat. Hazards Earth Syst. Sci., 19, 2887-2904, 2019

https://doi.org/10.5194/nhess-19-2887-2019-supplement

(C) Author(s) 2019. This work is distributed under

the Creative Commons Attribution 4.0 License.

(c) (1)

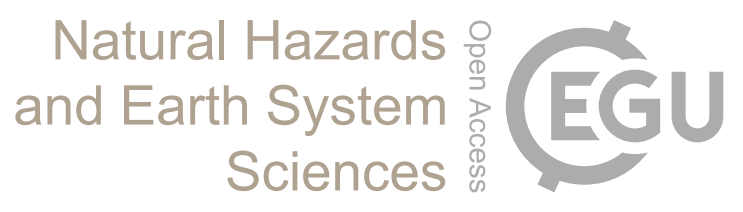

Supplement of

\title{
Tsunami risk perception in southern Italy: first evidence from a sample survey
}

Andrea Cerase et al.

Correspondence to: Andrea Cerase (andrea.cerase@uniroma1.it)

The copyright of individual parts of the supplement might differ from the CC BY 4.0 License. 


\section{단 \\ INGV}

\section{Survey on risk perception of tsunami of seismogenetic origin}

\section{Presentation Formula}

Good Morning, good evening, on behalf of the National Institute of Geophysics and Volcanology, we are carrying out a research on a phenomenon called tsunami. If you agree to answer, I will ask you some questions on this issue. The information we are going to collect will allow both the National Institute of Geophysics and Volcanology and the other institutions of the Civil Protection System to better understand the this type of phenomena is perceived by the population and thus prepare the most appropriate interventions.

Please note that the information you provide will not be associated in any way with your name, but will be treated anonymously and statistically aggregated. The interview will last a maximum of 10 minutes. Thank you for your availability

\section{SECTION 1: SOCIO-DEMOGRAPHIC DATA AND INFORMATION ON RESPONDENTS' BELONGING TERRITORY}

\section{Province}

Municipality

1) Gender $\square M \quad \square F \quad \square$ Not willing to declare it

\section{2) Age}

\section{3) Education}

Elementary licence

Middle school licence

$\checkmark$ Diploma of completion of secondary education

U University Degree

Master's degree, $\mathrm{PhD}$, other post-lauream titles

No qualification

\section{4) Nationality}

Italian

Non-Italian 
5) How is your family household composed? (multiple responses allowed)

I live alone

With adult(s)

With children under 6 years

With children under 6 years

With elders

With disabled / reduced mobility persons

6) How many kilometres far from the shore do you live?

Within $1 \mathrm{~km}$

From 1 to $3 \mathrm{~km}$

O Over $3 \mathrm{~km}$

- I don't know

7) How many generations is your family living on this coastline? (Even in different households in the same area)

-My family was here from before my great-grandparents

-My great-grandparents lived there

-My grandparents lived there

-My father or my mother lived there

$\square \mathrm{We}$ are the first generation settled here

SECTION 2: LEVEL OF AWARENESS AND SOURCES OF KNOWLEDGE ABOUT TSUNAMI HAZARD

8) Have you ever heard about tsunami?

$\square$ Yes $\square$ No

(if not, go to question 14.)

9) Can you tell me which words do you associate with tsunami?

DEarthquake; Dseaquake; Dlarge wave; Dport; Dflooding; Drough sea;

Dinundation; $\square$ sea withdrawal; $\square$ currents; $\square$ strong wind.

10) Can you tell me which words do you associate with seaquake?

DEarthquake; Useaquake; Dlarge wave; Dport; Dflooding; Drough sea;

Dinundation; Usea withdrawal; Dcurrents; Ustrong wind.

11) Can you tell me which word sound more familiar to you?

ITsunami USeaquake 
12)If you already heard about tsunami, from which sources? (multiple responses allowed)

Books

Newspapers

TV News

- Radio

$\square$ Internet

$\square$ Television programs / channels about science (SuperQuark, Focus, NatGeo etc.)

Movies

Civil Protection (\#loNonRischio - Maremoti, drills).

$\square$ Region, provinces or municipalities

$\square$ Research institutions / Universities

$\square$ Friends / relatives

DOther (specify)

Other

13)In the Mediterranean Sea the possibility of a tsunami / tidal wave is:

\begin{tabular}{|c|c|c|c|c|}
\hline $\begin{array}{c}\text { Not } \\
\text { probable }\end{array}$ & $\begin{array}{c}\text { Somewhat } \\
\text { improbable }\end{array}$ & $\begin{array}{c}\text { Neither } \\
\text { improbable } \\
\text { nor probable }\end{array}$ & $\begin{array}{c}\text { Somewhat } \\
\text { probable }\end{array}$ & $\begin{array}{c}\text { Very } \\
\text { probable }\end{array}$ \\
$\square$ & $\square$ & $\square$ & $\square$ & $\square$ \\
\hline
\end{tabular}

14)In your opinion, what are the causes that may trigger a tsunami / seaquake? (multiple responses allowed)

DEarthquakes

$\square$ Volcanic Eruptions

ULandslides

UMeteorites or asteroids at sea

uWeather phenomena

DOther (specify)

Other

I don't know 


\section{SECTION 3: CONTEXTUAL PERCEPTION OF TSUNAMI HAZARD}

15) The coast of your municipality are mainly
$\square$ Low and sandy
- High and sandy
$\square$ Low and rocky
$\square$ High and rocky
With inlets / coves
Without inlets / coves

16) Do you think that the coasts of your municipality / province can be affected by a tsunami / seaquake?
aYes a No
al don't know

17) In your opinion, which areas of the Mediterranean are more likely to be hit by tsunami / seaquake? (multiple responses allowed)

\begin{tabular}{|l|c|}
\hline $\begin{array}{l}\text { My own region or closer ones (Molise, Puglia, Calabria, Calabria, Sicily, } \\
\text { Campania, Lower Tyrrhenian Sea, Gulf of Taranto) }\end{array}$ & $\square$ \\
\hline Western Mediterranean (Spain, Morocco, Algeria) & $\square$ \\
\hline $\begin{array}{l}\text { Central Mediterranean (Italy, France, Slovenia, Croatia, Montenegro, } \\
\text { Bosnia Herzegovina, Albania, Greece, Tunisia, Malta, Libya) }\end{array}$ & $\square$ \\
\hline $\begin{array}{l}\text { Eastern Mediterranean (Coast Egypt, Israel, Lebanon, Syria, Turkey and } \\
\text { Cyprus)? }\end{array}$ & $\square$ \\
\hline I don't know & $\square$ \\
\hline
\end{tabular}

18) If a tsunami / seaquake would hit your region, how much do you think the water level could rise with respect to the normal sea level?

Less than $50 \mathrm{~cm}$

- Between $50 \mathrm{~cm}$ and 1 meter

a Between 1 meter and 3 meters

- Over 3 meters

a I don't know

19)If a tsunami / seaquake would hit your region, how far from the shore could the water arrive?

Less than 1 meter

a From 1 to 10 meters

- From 10 to 99 meters

- From hundred meters to kilometers

口 I don't know 
20)In your opinion, in case of tsunami / tsunami, to be dangerous for people near the shore how much should the water level with respect to the normal sea level?

- Less than $50 \mathrm{~cm}$

- Between $50 \mathrm{~cm}$ and 1 meter

- Between 1 meter and 3 meters

- Over 3 meters

I I don't know

21)What kind of phenomena may precede the arrival of a tsunami / seaquake? (How far do you agree with the following statements?)

A strong or long-lasting earthquake

\begin{tabular}{|c|c|c|c|c|}
\hline Strongly & Somewhat & Neither agree nor & Somewhat & Strongly \\
disagree & disagree & disagree & agree & Agree \\
$\square$ & $\square$ & $\square$ & $\square$ & $\square$ \\
\hline
\end{tabular}

Sea withdrawal

\begin{tabular}{|c|c|c|c|c|}
\hline Strongly & Somewhat & Neither agree nor & Somewhat & Strongly \\
disagree & disagree & disagree & agree & Agree \\
$\square$ & $\square$ & $\square$ & $\square$ & $\square$ \\
\hline
\end{tabular}

Sea level rise

\begin{tabular}{|c|c|c|c|c|}
\hline $\begin{array}{c}\text { Strongly } \\
\text { disagree }\end{array}$ & Somewhat & Neither agree nor & Somewhat & Strongly \\
$\square$ & $\square$ & disagree & agree & Agree \\
\hline
\end{tabular}

Strong sea currents

\begin{tabular}{|c|c|c|c|c|}
\hline Strongly & Somewhat & Neither agree nor & Somewhat & Strongly \\
disagree & disagree & disagree & agree & Agree \\
$\square$ & $\square$ & $\square$ & $\square$ & $\square$ \\
\hline
\end{tabular}

A strong and long-lasting uproar

\begin{tabular}{|c|c|c|c|c|}
\hline Strongly & Somewhat & Neither agree nor & Somewhat & Strongly \\
disagree & disagree & disagree & agree & Agree \\
$\square$ & $\square$ & $\square$ & $\square$ & $\square$ \\
\hline
\end{tabular}

22)Try to figure out the effects of a tsunami / tsunami on the coasts of your region. How far do you agree with the following statements?

Deaths or serious injuries

\begin{tabular}{|c|c|c|c|c|}
\hline $\begin{array}{c}\text { Strongly } \\
\text { disagree }\end{array}$ & $\begin{array}{c}\text { Somewhat } \\
\text { disagree }\end{array}$ & $\begin{array}{c}\text { Neither agree nor } \\
\text { disagree }\end{array}$ & Somewhat & Strongly \\
$\square$ & $\square$ & $\square$ & agree & Agree \\
& $\square$ & $\square$ & $\square$ \\
\hline
\end{tabular}


Damage / destruction of households, buildings or infrastructures

\begin{tabular}{|c|c|c|c|c|}
\hline Strongly & Somewhat & Neither agree nor & Somewhat & Strongly \\
disagree & disagree & disagree & agree & Agree \\
$\square$ & $\square$ & $\square$ & $\square$ & $\square$ \\
\hline
\end{tabular}

I lmpacts on natural environment (changes in coastal landscape, impacts on vegetation, crops, wild animals and livestock, etc.)

\begin{tabular}{|c|c|c|c|c|}
\hline Strongly & Somewhat & Neither agree nor & Somewhat & Strongly \\
disagree & disagree & disagree & agree & Agree \\
$\square$ & $\square$ & $\square$ & $\square$ & $\square$ \\
\hline
\end{tabular}

Impacts on the economy and work (destruction of coastal enterprises, damages to tourism industry, scarcity of financial resources, etc.)

\begin{tabular}{|c|c|c|c|c|}
\hline Strongly & Somewhat & Neither agree nor & Somewhat & Strongly \\
disagree & disagree & disagree & agree & Agree \\
$\square$ & $\square$ & $\square$ & $\square$ & $\square$ \\
\hline
\end{tabular}

\section{SECTION 4: REPRESENTATION OF TSUNAMIS}

\section{3) How far do you agree with the following statements?}

When you feel a strong earthquake near the coast one should flee to the beach

\begin{tabular}{|c|c|c|c|c|}
\hline $\begin{array}{c}\text { Strongly } \\
\text { disagree }\end{array}$ & $\begin{array}{c}\text { Somewhat } \\
\text { disagree }\end{array}$ & $\begin{array}{c}\text { Neither agree nor } \\
\text { disagree }\end{array}$ & $\begin{array}{c}\text { Somewhat } \\
\text { agree }\end{array}$ & $\begin{array}{c}\text { Strongly } \\
\text { Agree } \\
\square\end{array}$ \\
$\square$ & $\square$ & $\square$ & $\square$ \\
\hline
\end{tabular}

A tsunami waves can flood the coastal inlands for kilometers

\begin{tabular}{|c|c|c|c|c|}
\hline Strongly & Somewhat & Neither agree nor & Somewhat & Strongly \\
disagree & disagree & disagree & agree & Agree \\
$\square$ & $\square$ & $\square$ & $\square$ & $\square$ \\
\hline
\end{tabular}

A half-metre tsunami wave can drag an adult man into the sea

\begin{tabular}{|c|c|c|c|c|}
\hline Strongly & Somewhat & Neither agree nor & Somewhat & Strongly \\
disagree & disagree & disagree & agree & Agree \\
$\square$ & $\square$ & $\square$ & $\square$ & $\square$ \\
\hline
\end{tabular}

Sometimes tsunamis can be preceded by sea withdrawal, even for several tens of metres.

\begin{tabular}{|c|c|c|c|c|}
\hline Strongly & Somewhat & Neither agree nor & Somewhat & Strongly \\
disagree & disagree & disagree & agree & Agree \\
$\square$ & $\square$ & $\square$ & $\square$ & $\square$ \\
\hline
\end{tabular}


In the Mediterranean sea tsunami with waves higher than twenty meters are possible

\begin{tabular}{|c|c|c|c|c|}
\hline Strongly & Somewhat & Neither agree nor & Somewhat & Strongly \\
disagree & disagree & disagree & agree & Agree \\
$\square$ & $\square$ & $\square$ & $\square$ & $\square$ \\
\hline
\end{tabular}

\section{SECTION 5: CULTURAL ATTITUDES AND WORLDVISION}

\section{4) How far do you agree with the following statements?}

- To move on in life you have to work hard and do what you are told to do

\begin{tabular}{|c|c|c|c|c|}
\hline Strongly & Somewhat & Neither agree nor & Somewhat & Strongly \\
disagree & disagree & disagree & agree & Agree \\
$\square$ & $\square$ & $\square$ & $\square$ & $\square$ \\
\hline
\end{tabular}

When a natural disaster occurs, authorities should impose severe and immediate punishment on those who break the rules

\begin{tabular}{|c|c|c|c|c|}
\hline Strongly & Somewhat & Neither agree nor & Somewhat & Strongly \\
disagree & disagree & disagree & agree & Agree \\
$\square$ & $\square$ & $\square$ & $\square$ & $\square$ \\
\hline
\end{tabular}

The healthiest society is the one based on competition between individuals

\begin{tabular}{|c|c|c|c|c|}
\hline Strongly & Somewhat & Neither agree nor & Somewhat & Strongly \\
disagree & disagree & disagree & agree & Agree \\
$\square$ & $\square$ & $\square$ & $\square$ & $\square$ \\
\hline
\end{tabular}

To deal with natural disasters individuals should prepare themselves before instead of waiting for someone to help them after

\begin{tabular}{|c|c|c|c|c|}
\hline Strongly & Somewhat & Neither agree nor & Somewhat & Strongly \\
disagree & disagree & disagree & agree & Agree \\
$\square$ & $\square$ & $\square$ & $\square$ & $\square$ \\
\hline
\end{tabular}

Many conflicts could be resolved by a fairer division of workload and natural resources

\begin{tabular}{|c|c|c|c|c|}
\hline Strongly & Somewhat & Neither agree nor & Somewhat & Strongly \\
disagree & disagree & disagree & agree & Agree \\
$\square$ & $\square$ & $\square$ & $\square$ & $\square$ \\
\hline
\end{tabular}

Natural disasters do not exist: it is only nature offended by man's arrogance and greediness, that rebels

\begin{tabular}{|c|c|c|c|c|}
\hline Strongly & Somewhat & Neither agree nor & Somewhat & Strongly \\
disagree & disagree & disagree & agree & Agree \\
$\square$ & $\square$ & $\square$ & $\square$ & $\square$ \\
\hline
\end{tabular}

When you do something for others you should not expect gratefulness

\begin{tabular}{|c|c|c|c|c|}
\hline $\begin{array}{c}\text { Strongly } \\
\text { disagree }\end{array}$ & $\begin{array}{c}\text { Somewhat } \\
\text { disagree }\end{array}$ & $\begin{array}{c}\text { Neither agree nor } \\
\text { disagree }\end{array}$ & $\begin{array}{c}\text { Somewhat } \\
\text { agree }\end{array}$ & $\begin{array}{c}\text { Strongly } \\
\text { Agree } \\
\square\end{array}$ \\
$\square$ & $\square$ & $\square$ & $\square$ \\
\hline
\end{tabular}


- Natural disasters serve to remind us that making plans is useless: our lives are determined by forces that we cannot control

\begin{tabular}{|c|c|c|c|c|}
\hline $\begin{array}{c}\text { Strongly } \\
\text { disagree }\end{array}$ & $\begin{array}{c}\text { Somewhat } \\
\text { disagree }\end{array}$ & $\begin{array}{c}\text { Neither agree nor } \\
\text { disagree }\end{array}$ & $\begin{array}{c}\text { Somewhat } \\
\text { agree }\end{array}$ & Agrongly \\
$\square$ & $\square$ & $\square$ & $\square$ & $\square$ \\
\hline
\end{tabular}

\section{SECTION 6: MESSAGES AND CHANNELS TO SPREAD TSUNAMI ALERT}

25) In your opinion, is it possible to spread an early warning before the arrival of a tsunami on the way to the coast?

Y Yes $\square$ No $\square$ I don't know (If answer is "not" or "I don't know" go to 25)

26) In your opinion, who should warn population about the impending arrival of a tsunami on the way to the coast?

1.

2.

3.

27) If a tsunami would hit your region's coastline, which channels would you prefer to receive an early warning? (max three answers)

\begin{tabular}{|l|}
\hline$\square$ Television \\
\hline Radio \\
\hline$\square$ Internet and social media \\
\hline$\square$ E-mail \\
\hline$\square$ Phone call (mobile or fixed telephone) \\
\hline$\square$ Be advised by friends, neighbours or relatives \\
\hline$\square$ SMS \\
\hline$\square$ Smarthpone application from official sources \\
\hline$\square$ Sirens or other acoustic devices \\
\hline
\end{tabular}




\title{
. \\ INGV
}

\section{Indagine sulla percezione del rischio da tsunami / maremoto di origine sismogenetica}

\begin{abstract}
Formula di presentazione
Buongiorno/buonasera, per conto dell'Istituto Nazionale di Geofisica e Vulcanologia stiamo svolgendo una ricerca su un fenomeno chiamato tsunami o maremoto. Se accetta di rispondere, le farò alcune domande su questo tema. Le informazioni raccolte permetteranno all'lstituto Nazionale di Geofisica e Vulcanologia e alle altre istituzioni del Sistema di Protezione Civile di conoscere meglio la percezione di questo tipo di fenomeni da parte della popolazione e predisporre cosi gli interventi più adeguati.

Le ricordo che le informazioni da lei fornite non saranno in alcun modo associate al suo nominativo ma saranno trattate in forma anonima e aggregate statisticamente. L'intervista durerà al massimo 10 minuti. La ringrazio per la sua disponibilità
\end{abstract}

\section{SEZIONE 1: DATI SOCIO-DEMOGRAFICI E INFORMAZIONI SUL TERRITORIO} D'APPARTENENZA

\section{Provincia}

Comune di residenza

1) Genere $\square M \square F \square$ Preferisco non dichiararlo

\section{2) Età}

\section{3) Titolo di studio}

$\square$ Licenza elementare

Licenza media inferiore

$\square$ Diploma media superiore

Laurea

Dottorato, Master o altro titolo post-laurea

$\square$ Nessun titolo di studio

\section{4) Nazionalità}

I Italiana

Straniera 
5) Come è composto il suo nucleo familiare (sono possibili più risposte)

vivo da solo/a

con adulti

con bambini inferiori a 6 anni

$\square$ con bambini maggiori di 6 anni

$\square$ con anziani

con persone con disabilità 0 a mobilità ridotta

6) A quanti chilometri di distanza dalla costa vive?

entro $1 \mathrm{~km}$

da 1 a $3 \mathrm{~km}$

Oltre $3 \mathrm{~km}$

Non saprei

7) Da quante generazioni la sua famiglia risiede in questo tratto di costa? (Anche se in altre abitazioni)

- La mia famiglia era qui da prima dei miei bisnonni

Ci abitavano i miei bisnonni

$\checkmark$ Ci abitavano i miei nonni

Ci abitavano mio padre o mia madre

$\checkmark$ Siamo la prima generazione che si è stabilita qui

SEZIONE 2: LIVELLO DI CONOSCENZA E FONTI DINNFORMAZIONE SUL RISCHIO TSUNAMI

8) Ha mai sentito parlare di tsunami o maremoto?

¿ Si $\square$ No

9) Quali tra le seguenti parole associa a Tsunami:

Dterremoto; Imareggiata; Dgrande onda; Dporto; Dallagamento;

Dinondazione; $\square$ ritiro delle acque; I mare mosso; $\square$ correnti ; $\square$ forte vento: $\square$ altro

10)Quali tra le seguenti parole associa a Maremoto:

Dterremoto; $\square$ mareggiata; Jgrande onda; Dporto; Dallagamento;

Dinondazione; Dritiro delle acque; $\square$ mare mosso; $\square$ correnti ; $\square$ forte vento; $\square$ altro

11) Può indicarmi quale parola le risulta più familiare?

口Tsunami aMaremoto (da qui in poi l'intervistatore usa SOLO tsunami o maremoto) 
12)Se ha già sentito parlare di tsunami/maremoto da quali fonti? (possibili più risposte)

प Libri

G Giornali

$\square$ Telegiornali

$\square$ Radio

Internet

Programmi o canali scientifici (SuperQuark, Focus, NatGeo etc.)

Gilm

Protezione Civile (campagna \#loNonRischio - Maremoto, esercitazioni etc).

Regione, provincia o comune

Enti di ricerca o Università

Amici e familiari

Altro (specificare)

13) Nel Mar Mediterraneo la possibilità che si verifichi uno tsunami / maremoto è:

\begin{tabular}{|c|c|c|c|c|}
\hline $\begin{array}{c}\text { Per niente } \\
\text { probabile }\end{array}$ & Poco & Né probabile né & Abbastanza & Molto probabile \\
$\square$ & $\square$ & improbabile & probabile & $\square$ \\
\hline
\end{tabular}

14)Secondo lei, quali sono le cause che generano uno tsunami / maremoto?

(Possibili più risposte)

- Terremoti

Eruzioni vulcaniche

Grane

Meteoriti $o$ asteroidi in mare

- Fenomeni meteo

Altro (specificare)

Non saprei

SEZIONE 3: LA PERCEZIONE CONTESTUALE DEL RISCHIO TSUNAMI

15) Le coste del suo comune sono prevalentemente?

Basse e sabbiose

Basse e rocciose

$\square$ Con la presenza di insenature
Alte e sabbiose

Alte e rocciose

$\square$ Senza insenature

16)Pensa che le coste del Comune (o della sua provincia) in cui vive possano essere colpite da uno tsunami / maremoto?

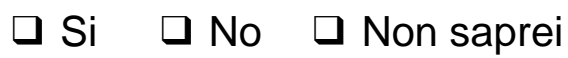


17)Secondo lei, qual è la zona del Mediterraneo che ha una più alta probabilità di essere colpita da uno tsunami / maremoto?

\begin{tabular}{|l|c|}
\hline $\begin{array}{l}\text { La mia regione o quelle vicine (Molise, Puglia, Calabria, Sicilia, Campania, Basso } \\
\text { Tirreno, golfo di Taranto) }\end{array}$ & $\square$ \\
\hline II Mediterraneo Occidentale (Coste Spagna, Marocco, Algeria, Nord Africa) & $\square$ \\
\hline II Mediterraneo Centrale (Coste Italia, Francia, Slovenia, Croazia, Montenegro, \\
Bosnia Erzegovina, Albania, Grecia, Tunisia, Malta, Libia) & $\square$ \\
\hline II Mediterraneo orientale (Coste Egitto, Israele, Libano, Siria, Turchia e Cipro) & $\square$ \\
\hline Non saprei & $\square$ \\
\hline
\end{tabular}

18)Se uno tsunami / maremoto dovesse colpire la costa del suo comune, di quanto pensa possa salire il livello dell'acqua rispetto al normale livello del mare?

- Meno di 50 centimetri

- Tra 50 centimetri e 1 metro

- Tra 1 metro e 3 metri

Oltre 3 metri

Non saprei

19) Se un'onda di tsunami / maremoto dovesse colpire la costa del suo comune, fino a che distanza dalla riva potrebbe arrivare l'acqua?

Meno di qualche metro

Da 1 a 10 metri

Q Qualche decina di metri

Da centinaia di metri a chilometri

Non saprei

20)Secondo lei, in caso di tsunami / maremoto, di quanto deve innalzarsi il livello dell'acqua rispetto al normale livello del mare, per essere pericoloso per le persone che si trovano in prossimità della riva?

- Meno di 50 centimetri

- Tra 50 centimetri e 1 metro

- Tra 1 metro e 3 metri

- Oltre 3 metri

- Non saprei 
21)Quali fenomeni possono precedere l'arrivo di uno tsunami / maremoto? (Può indicarci il grado di accordo con le seguenti affermazioni?)

Un forte terremoto

\begin{tabular}{|c|c|c|c|c|}
\hline $\begin{array}{c}\text { Per niente } \\
\text { d'accordo }\end{array}$ & Poco & Né d'accordo né & Abbastanza & Molto d'accordo \\
in disaccordo & d'accordo & \\
$\square$ & $\square$ & $\square$ & $\square$ & $\square$ \\
\hline
\end{tabular}

II mare che si ritira

\begin{tabular}{|c|c|c|c|c|}
\hline $\begin{array}{c}\text { Per niente } \\
\text { d'accordo }\end{array}$ & Poco & Né d'accordo né & Abbastanza & Molto d'accordo \\
in disaccordo & d'accordo & \\
$\square$ & $\square$ & $\square$ & $\square$ & $\square$ \\
\hline
\end{tabular}

Un innalzamento del livello del mare

\begin{tabular}{|c|c|c|c|c|}
\hline $\begin{array}{c}\text { Per niente } \\
\text { d'accordo }\end{array}$ & Poco & Né d'accordo né & Abbastanza & Molto d'accordo \\
in disaccordo & d'accordo & \\
$\square$ & $\square$ & $\square$ & $\square$ & $\square$ \\
\hline
\end{tabular}

Forti correnti marine

\begin{tabular}{|c|c|c|c|c|}
\hline $\begin{array}{c}\text { Per niente } \\
\text { d'accordo }\end{array}$ & Poco & Né d'accordo né \\
in disaccordo & Abbastanza \\
d'accordo & Molto d'accordo & \\
$\square$ & $\square$ & $\square$ & $\square$ \\
\hline
\end{tabular}

Un rumore forte e prolungato

\begin{tabular}{|c|c|c|c|c|}
\hline $\begin{array}{c}\text { Per niente } \\
\text { d'accordo } \\
\square\end{array}$ & $\begin{array}{c}\text { Poco } \\
\text { d'accordo }\end{array}$ & $\begin{array}{c}\text { Né d'accordo né } \\
\text { in disaccordo }\end{array}$ & $\begin{array}{c}\text { Abbastanza } \\
\text { d'accordo } \\
\square\end{array}$ & $\begin{array}{c}\text { Molto d'accordo } \\
\square\end{array}$ \\
\hline
\end{tabular}

22)Provi a immaginare gli effetti di uno tsunami / maremoto sulle coste della sua regione. Può indicarci il grado di accordo con le seguenti affermazioni?

Persone morte o seriamente ferite

\begin{tabular}{|c|c|c|c|c|}
\hline $\begin{array}{c}\text { Per niente } \\
\text { d'accordo }\end{array}$ & Poco & Né d'accordo né & Abbastanza & Molto d'accordo \\
in disaccordo & d'accordo \\
$\square$ & $\square$ & $\square$ & $\square$ & $\square$ \\
\hline
\end{tabular}

Danni/ distruzioni ad abitazioni, edifici o infrastrutture

\begin{tabular}{|c|c|c|c|c|}
\hline $\begin{array}{l}\text { Per niente } \\
\text { d'accordo }\end{array}$ & $\begin{array}{l}\text { Poco } \\
\text { d'accordo } \\
\end{array}$ & $\begin{array}{l}\text { Né d'accordo né } \\
\text { in disaccordo }\end{array}$ & $\begin{array}{c}\text { Abbastanza } \\
\text { d'accordo } \\
\end{array}$ & $\begin{array}{c}\text { Molto d'accordo } \\
\square\end{array}$ \\
\hline
\end{tabular}

Impatti sull'ambiente naturale (cambiamento del paesaggio costiero, impatto sulle piante e sulle coltivazioni, impatto sugli animali, etc.)

\begin{tabular}{|c|c|c|c|c|}
\hline $\begin{array}{c}\text { Per niente } \\
\text { d'accordo } \\
\square\end{array}$ & $\begin{array}{c}\text { Poco } \\
\text { d'accordo } \\
\square\end{array}$ & $\begin{array}{c}\text { Né d'accordo né } \\
\text { in disaccordo } \\
\square\end{array}$ & $\begin{array}{c}\text { Abbastanza } \\
\text { d'accordo }\end{array}$ & Molto d'accordo \\
\hline
\end{tabular}


Impatti sull'economia e sul lavoro (distruzione delle imprese sulla costa, danni al turismo, scarsità di risorse finanziarie, etc.)

\begin{tabular}{|c|c|c|c|c|}
\hline $\begin{array}{c}\text { Per niente } \\
\text { d'accordo }\end{array}$ & Poco & Né d'accordo né & Abbastanza & Molto d'accordo \\
in disaccordo & d'accordo & \\
$\square$ & $\square$ & $\square$ & $\square$ & $\square$ \\
\hline
\end{tabular}

\section{SEZIONE 4: LE RAPPRESENTAZIONI DEGLI TSUNAMI}

\section{3)Può indicarci il grado di accordo con le seguenti affermazioni?}

Se ci si trova vicino alla costa e si avverte un forte terremoto bisogna fuggire verso la spiaggia

\begin{tabular}{|c|c|c|c|c|}
\hline $\begin{array}{c}\text { Per niente } \\
\text { d'accordo } \\
\square\end{array}$ & $\begin{array}{c}\text { Poco } \\
\text { d'accordo }\end{array}$ & $\begin{array}{c}\text { Né d'accordo né } \\
\text { in disaccordo }\end{array}$ & $\begin{array}{c}\text { Abbastanza } \\
\text { d'accordo }\end{array}$ & Molto d'accordo \\
\hline
\end{tabular}

Gli tsunami / maremoti possono inondare l'entroterra costiero per una distanza di chilometri

\begin{tabular}{|c|c|c|c|c|}
\hline $\begin{array}{c}\text { Per niente } \\
\text { d'accordo } \\
\square\end{array}$ & $\begin{array}{c}\text { Poco d'accordo } \\
\square\end{array}$ & $\begin{array}{c}\text { Né d'accordo né } \\
\text { in disaccordo }\end{array}$ & $\begin{array}{c}\text { Abbastanza } \\
\text { d'accordo }\end{array}$ & $\begin{array}{c}\text { Molto d'accordo } \\
\end{array}$ \\
\hline
\end{tabular}

Un'onda di tsunami / maremoto di mezzo metro può trascinare in mare un uomo adulto

\begin{tabular}{|c|c|c|c|c|}
\hline $\begin{array}{c}\text { Per niente } \\
\text { d'accordo }\end{array}$ & $\begin{array}{c}\text { Poco } \\
\text { d'accordo }\end{array}$ & $\begin{array}{c}\text { Né d'accordo né } \\
\text { in disaccordo }\end{array}$ & $\begin{array}{c}\text { Abbastanza } \\
\text { d'accordo }\end{array}$ & Molto d'accordo \\
\hline & $\square$ & $\square$ & $\square$ & $\square$ \\
\hline
\end{tabular}

A volte gli tsunami / maremoti possono essere preceduti dal ritirarsi del mare, per alcune decine di metri o anche di più

\begin{tabular}{|c|c|c|c|c|}
\hline $\begin{array}{c}\text { Per niente } \\
\text { d'accordo }\end{array}$ & Poco \\
$\square$ & d'accordo & $\begin{array}{c}\text { Né d'accordo né } \\
\text { in disaccordo }\end{array}$ & $\begin{array}{c}\text { Abbastanza } \\
\text { d'accordo } \\
\square\end{array}$ & Molto d'accordo \\
\hline
\end{tabular}

Nel Mar Mediterraneo sono possibili tsunami / maremoti con onde alte anche più di venti metri

\begin{tabular}{|c|c|c|c|c|}
\hline $\begin{array}{c}\text { Per niente } \\
\text { d'accordo } \\
\square\end{array}$ & P'accordo & $\begin{array}{c}\text { Né d'accordo né } \\
\text { in disaccordo } \\
\square\end{array}$ & $\begin{array}{c}\text { Abbastanza } \\
\text { d'accordo }\end{array}$ & Molto d'accordo \\
\hline
\end{tabular}


SEZIONE 5: ATTEGGIAMENTI CULTURALI E VISIONI DEL MONDO

\section{4)Può indicarci il grado di accordo con le seguenti affermazioni?}

Per andare avanti nella vita bisogna lavorare duramente e fare ciò che ti viene detto di fare

\begin{tabular}{|c|c|c|c|c|}
\hline $\begin{array}{c}\text { Per niente } \\
\text { d'accordo }\end{array}$ & $\begin{array}{c}\text { Poco } \\
\text { d'accordo }\end{array}$ & $\begin{array}{c}\text { Né d'accordo } \\
\text { né in } \\
\text { disaccordo } \\
\square\end{array}$ & $\begin{array}{c}\text { Abbastanza } \\
\text { d'accordo }\end{array}$ & Molto d'accordo \\
& $\square$ & $\square$ & $\square$ \\
\hline
\end{tabular}

Quando accade un disastro naturale le autorità dovrebbero imporre punizioni severe e immediate a chi infrange le regole

\begin{tabular}{|c|c|c|c|c|}
\hline $\begin{array}{c}\text { Per niente } \\
\text { d'accordo }\end{array}$ & Poco & Né d'accordo & Abbastanza \\
d'accordo & né in & Molto d'accordo \\
disaccordo & & \\
\hline
\end{tabular}

La società più sana è quella che si regge sulla competizione tra gli individui

\begin{tabular}{|c|c|c|c|c|}
\hline $\begin{array}{c}\text { Per niente } \\
\text { d'accordo }\end{array}$ & Poco & Né d'accordo & Abbastanza & Molto d'accordo \\
d'accordo & né in & disaccordo & & \\
\hline & $\square$ & $\square$ & $\square$ \\
\hline
\end{tabular}

Per affrontare i disastri naturali gli individui dovrebbero prepararsi prima invece di aspettare che qualcuno li aiuti dopo

\begin{tabular}{|c|c|c|c|c|}
\hline $\begin{array}{c}\text { Per niente } \\
\text { d'accordo }\end{array}$ & $\begin{array}{c}\text { Poco } \\
\text { d'accordo }\end{array}$ & $\begin{array}{c}\text { Né d'accordo } \\
\text { né in } \\
\text { disaccordo }\end{array}$ & $\begin{array}{c}\text { Abbastanza } \\
\text { d'accordo }\end{array}$ & $\begin{array}{c}\text { Molto d'accordo } \\
\square\end{array}$ \\
$\square$ & $\square$ & $\square$ & $\square$ \\
\hline
\end{tabular}

Molti conflitti si potrebbero risolvere con una più giusta divisione del lavoro e delle risorse naturali disponibili

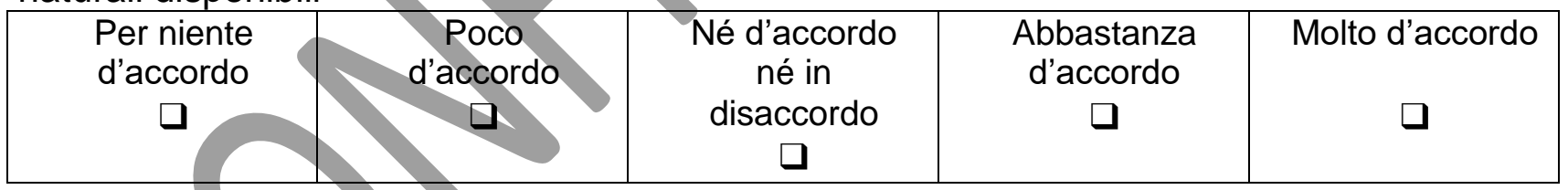

I disastri naturali non esistono: è soltanto la natura che si ribella all'arroganza e all'avidità dell'uomo

\begin{tabular}{|c|c|c|c|c|}
\hline $\begin{array}{c}\text { Per niente } \\
\text { d'accordo }\end{array}$ & Poco & $\begin{array}{c}\text { Né d'accordo } \\
\text { né in } \\
\text { d'accordo }\end{array}$ & $\begin{array}{c}\text { Abbastanza } \\
\text { d'accordo }\end{array}$ & Molto d'accordo \\
& $\square$ & $\square$ & $\square$ \\
\hline
\end{tabular}

Nella vita se si fa qualcosa per gli altri non bisogna aspettarsi riconoscenza e gratitudine

\begin{tabular}{|c|c|c|c|c|}
\hline $\begin{array}{c}\text { Per niente } \\
\text { d'accordo }\end{array}$ & $\begin{array}{c}\text { Poco } \\
\text { d'accordo }\end{array}$ & $\begin{array}{c}\text { Né d'accordo } \\
\text { né in } \\
\text { disaccordo }\end{array}$ & $\begin{array}{c}\text { Abbastanza } \\
\text { d'accordo }\end{array}$ & Molto d'accordo \\
& $\square$ & $\square$ & $\square$ \\
\hline
\end{tabular}


I disastri naturali servono a ricordarci che fare piani è inutile: le nostre vite sono determinate da forze che non siamo in grado di controllare

\begin{tabular}{|c|c|c|c|c|}
\hline $\begin{array}{c}\text { Per niente } \\
\text { d'accordo }\end{array}$ & Poco & Né d'accordo & Abbastanza \\
d'accordo & né in \\
disaccordo & $\square$ & $\square$ & $\square$ \\
\hline
\end{tabular}

\section{SEZIONE 6: MESSAGGI E CANALI PER L'ALLERTA TSUNAMI}

25) Secondo lei è possibile avvertire le popolazioni italiane dell'imminente arrivo di uno tsunami / maremoto sulle coste?

$\square \mathrm{Si} \quad \mathrm{No} \square$ Non saprei (se rispondono no o non saprei vai alla 25)

26) Secondo lei chi dovrebbe avvertire le popolazioni dell'imminente arrivo sulle coste di uno tsunami / maremoto?

1.

2.

3.

27) In che modo preferirebbe essere avvertito dell'imminente arrivo di uno tsunami/maremoto? (massimo tre risposte)

\begin{tabular}{|l|}
\hline$\square$ Televisione \\
\hline Radio \\
\hline Internet e social media \\
\hline E-mail \\
\hline Telefonata su telefono fisso o cellulare \\
\hline A voce da amici, vicini o parenti \\
\hline SMS \\
\hline App per smartphone di una fonte ufficiale \\
\hline Segnali acustici (sirene) \\
\hline
\end{tabular}

